\section{Therapeutic Ultrasound in Dentistry \\ Applications for Dentofacial Repair, Regeneration, and Tissue Engineering}

Editors: Tarek El-Bialy, Eiji Tanaka, Dror

Aizenbud

Publisher: Springer International

Publishing AG, Germany

Language: English

ISBN: 978-3-319-66322-7

Edition: 1/e

Publish Year: 2018

Pages: 96 , illustrated

Price: $117,69 €$

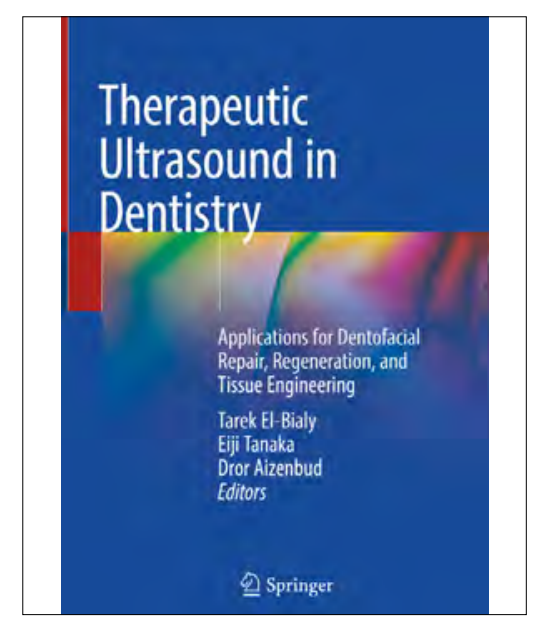

This book highlights a new possibility of dental treatment for some pathological conditions considered untreatable with conventional methods by the use of low-intensity pulsed ultrasound (LIPUS). The book is divided in 11 chapters. A series of information on LIPUS aspects, how to act and the biological mechanisms on dentofacial bioengineering are presented. The role of LIPUS for bone healing, skeletal muscles, periodontal tissue regeneration, temporomandibular joints and salivary glands is convincingly explained in a number of chapters. LIPUS application in orthodontics, dentofacial region, shockwave therapy in orthodontics, and pulp cell differentiation and future directions are the subjects of other chapters.

The authors, presenting and illustrating the latest discoveries and uses of ultrasound in dentistry, manage to attain their aim and write a particularly useful book for dental surgeons, orthodontists, periodontologists, and other practitioners. 